\title{
Serum Cortisol and Urinary Vanillyl Mandelic Acid Levels in Critically Ill Children R.S.Arafa, Y.M.Ismail, E.H.Assar and S.H.Hafez
}

Paediatrics Dept., Faculty of Medicine, Benha Univ., Benha, Egypt

E-Mail:saramh.12@gmail.com

\begin{abstract}
The term fundamentally sick youngster doesn't allude to a specific illness yet it alludes to any kid who is in a clinical state which may bring about respiratory or heart failure or cut off neurologic complexities if not perceived and treated expeditiously. The point of this postulation wass to decide the scope of serum cortisol and urinary Vanillyl Mandelic Acid (VMA) levels in a gathering of basically sick kids and correspond them to seriousness and result. This cross sectional examination included 40 pediatric patients going from 1month to $18 y e a r s$ Acute Physiology and Chronic Health Evaluation II(APACHE II) scoring framework which depends on deviations from typical of 11acute physiologic variablesAll patients had been researched for Assessment of cortisol level (ELISA) and VMA leve. Mean APACHE II score was essentially higher in non-survivors (20) contrasted with 12 in non-survivors. Middle Cortisol level was essentially higher in nonsurvivors (50) contrasted with survivors (20). Middle VMA was altogether higher in non-survivors (41) contrasted with survivors (10). Cortisol and VMA levels are raised in non-survivors contrasted with survivors. We recommend that the accompanying admonitions be borne as a main priority when diagnosing adrenal inadequacy in the basically sick patient.
\end{abstract}

\section{Introduction}

The solid connection among stress and adrenocortical capacity was first depicted in 1923 by Scott and afterward was explored in detail by Selye. From that point forward, it is all around acknowledged that pressure, for example, consumes, medical procedure, sepsis and basic ailment, is related with raised cortisol levels and catecholamines basically epinephrine and nor epinephrine. This reaction is essential for guideline of homeostasis and survival.Later human examinations showed the association between the seriousness of stress and the extent of adrenocortical reaction $[1,2]$.

Cortisol is a steroid chemical created by the adrenal glands.Its work is to build glucose, smother the resistant framework, help in fat, protein and starch digestion, keep up vascular tone and endothelial uprightness and potentiate catecholamine activities [3].

Then again, epinephrine is a chemical and a synapse. It builds pulse, chokes veins, widens air sections, and partakes in battle or flight reaction of the thoughtful sensory system. Vanillyl Mandelic Acid (VMA) is the urinary metabolite of epinephrine and other catecholamine's catabolism so the degree of VMA can be utilized as marker of catecholamines level, so the thoughtful action can be checked with pee Vanillyl Mandelic Acid [4] .

Because of restricted wellbeing sources and an expansion in the expense of wellbeing the executives, visualization from the illness has become avery significant territory of wellbeing sciences. Numerous significant scoring frameworks have been produced for escalated care units. These scoring frameworks give net gauge of mortality hazards in concentrated consideration units patients. Quite possibly the most every now and again utilized scoring frameworks is APACHE-II (intense physiology and ongoing wellbeing assessment II). The APACHE-II score comprises of three segments. The biggest part of the APACHE-II score is Acute Physiology Score (APS), got from 12 clinical estimations that are acquired inside 24 hours after admission to the ICU [5].
The most anomalous estimation is chosen to create the APS segment of the APACHE-II score. On the off chance that variable has not been estimated, it is allocated zero focuses. The factors are inside temperature, pulse, mean blood vessel pressure, respiratory rate, oxygenation, blood vessel $\mathrm{pH}$, serum sodium, serum potassium, serum creatinin, haematocrit, white platelets tally and Glasgow extreme lethargies scale. Second part is age change: From one to six focuses are added for patients more seasoned than 44 years old. Third segment of APACHE-II is persistent wellbeing assessment. An extra change is made for patients with extreme and persistent organ disappointment including the heart, lungs, kidneys, liver and safe framework [5].

The point of this proposition was to decide the scope of serum cortisol and urinary Vanillyl mandelic corrosive (VMA) levels in a gathering of fundamentally sick kids and correspond them to seriousness and result.

\section{Patient and Method}

This cross sectional study involved 40 pediatric patients ranging from 1 month to 18 years

\subsection{Inclusion criteria}

- The age is between 1 month and 18 years.

- Both sexes are included.

- Children admitted in intensive care unit.

\section{Exclusion criteria}

- Any patient takes medications that could affect Vanillyl Mandelic Acid measurments, such as reserpine,morphine and pentobarbital.

- Any patient takes medications that could affect cortisol measurments, such as corticosteroids or systemic treatment with imidazoles.

- Any patient with endocrinal disorders.

- The severity of the disease will be assessed with in $24 \mathrm{~h}$ after admission by means of the original Acute Physiology and Chronic Health Evaluation II(APACHE II) scoring system which is based on deviations from normal of 11acute physiologic 
variables, the Glasgow Coma Scale (GCS), the age of the patient,and the presence or absence of chronic disease. Because the frequent use of drugs for sedation makes the GCS unreliable, we also determined at the same time a modified

- APACHE II score (APACHE IIm), which is defined as the original APACHE II score without the GCS.

- All patients had been investigated for Assessment of cortisol level (ELISA) and VMA level.

\section{Results}

Mean time of study populace was 6.3 years with standard deviation of 3 years.As respect sexual orientation, $67.5 \%$ were guys while $32.5 \%$ were females. The most incessant instance of affirmation was respiratory trouble $(27.5 \%)$ trailed by gastroenteritis with extreme parchedness $(17.5 \%)$ at that point postoperative causes $(15.0 \%)$. As respect cortisol, middle level was 27 and gone from 1 to 80 .

As respect VMA, middle level was 12.5 and gone from 3 to $101 \mathrm{Mean}$ APACHE II score was 13 with standard deviation of 6 . Middle mortality hazard was 15 and gone from 1 to $40.12 .5 \%$ of study populace kicked the bucket. Mean PICU stay was 7 days with standard deviation of 3 . Cortisol demonstrated non-huge relationships with other examination boundaries.

VMA demonstrated huge positive relationship with APACHE II score $(r=0.395$ and $P$ esteem $=0.012)$ There were no huge connections between's VMA and different boundaries There were no huge contrasts between survivors and non-survivors as respect age and sex. $\mathrm{P}$ esteems were 0.843 and 0.307 Mean APACHE II score was fundamentally higher in non-survivors (20) contrasted with 12 in non-survivors. $P$ esteem was 0.002 mMedian mortality hazard was higher in nonsurvivors (40) contrasted with 15 in survivors. P esteem was 0.012 Median Cortisol level was fundamentally higher in non-survivors (50) contrasted with survivors (20). P esteem was 0.042 Median VMA was fundamentally higher in non-survivors (41) contrasted with survivors (10). P esteem was 0.021

ROC examination was accomplished for forecast of mortality. It was discovered that AUC was 0.783 with $95 \%$ CI going from 0.625 to 0.940 . Best cutoff was $>29$ at which affectability and explicitness were $100 \%$ and $60 \%$ individually.

Table (1) APACHE score and mortality risk in survivors and non-survivors.

\begin{tabular}{|c|c|c|c|c|}
\hline & \multicolumn{4}{|c|}{ Mortality } \\
\hline & & Yes & No & P value \\
\hline APACHE II score & Mean \pm SD & $20 \pm 4$ & $12 \pm 5$ & 0.005 \\
\hline Mortality risk (\%) & Median (range) & $40(15-40)$ & $15(1-40)$ & 0.012 \\
\hline
\end{tabular}

Mann Whitney U test was used.

Table (2) Cortisol \& VMA levels in survivors and non-survivors.

\begin{tabular}{lcccc}
\hline \multicolumn{4}{c}{ Mortality } \\
\hline Cortisol & Mes & No & P value \\
VMA & Median (range) & $50(29.83-79)$ & $20(1-80)$ & 0.042 \\
\hline
\end{tabular}

Mann Whitney U test was used.

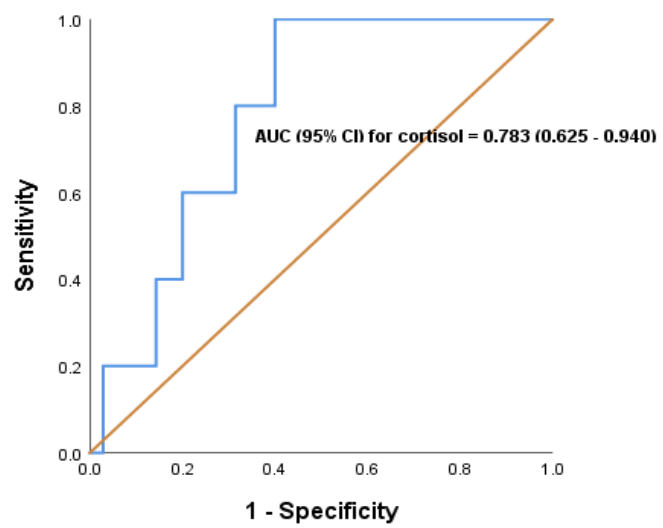

Fig (1) ROC analysis for Cortisol in prediction of mortality. 


\section{Discussion}

The determination of adrenal deficiency (AI) is quite possibly the most questionable points in basic consideration medication. Clinically evident AI is unprecedented in fundamentally sick patients and AI or basic ailment related corticosteroid deficiency is characterized as a deficient glucocorticoid reaction, estimated by the pinnacle cortisol or the augmentation in the cortisol level after exogenous corticotropin (ACTH) organization [6].

The current examination demonstrated that as respect reason for affirmation, the most regular instance of confirmation was respiratory misery $(27.5 \%)$ trailed by gastroenteritis with extreme lack of hydration (17.5\%) at that point postoperative causes $(15.0 \%)$.

In the investigation of Balbão et al., [2], the demonstrative classes were sepsis/septic stun $(\mathrm{n}=19$; $56 \%)$, intense respiratory disappointment $(\mathrm{n}=10 ; 29 \%)$, significant consume $(\mathrm{n}=4 ; 12 \%)$ and horrible mind injury $(n=1 ; 3 \%)$.

Supreme adrenocortical deficiency (analyzed by low plasma cortisol fixations) is exceptional in the escalated care populace. The finding of relative adrenocortical inadequacy (for example raised basal plasma cortisol levels with an odd expansion in plasma focuses following an ACTH upgrade) keeps on producing a lot of discussion. Regardless of numerous distributions regarding this matter throughout the most recent fifty years, this subject keeps on being hounded by debate. A raised complete plasma cortisol in focused on ICU patients has been shown in various examinations [7].

Like pheochromocytomas, neuroblastomas produce catecholamines however have a restricted limit with regards to catecholamine stockpiling and emission so the catecholamines created are to a great extent processed inside tumor cells. Therefore, biochemical testing for neuroblastoma has depended on estimations of homovanillic corrosive (HVA) and vanillylmandelic corrosive (VMA). As the last final results of catecholamine digestion, these metabolites are discharged in pee at micromolar fixations, guaranteeing their simple estimation and proceeded with use for biochemical testing [8].

The current investigation indicated that as respect cortisol, middle level was 27 and gone from 1 to 80 . As respect VMA, middle level was 12.5 and gone from 3 to 101.

The current investigation indicated that mean APACHE II score was 13 with standard deviation of 6 . Middle mortality hazard was 15 and gone from 1 to 40 . $12.5 \%$ of study populace passed on. Mean PICU stay was 7 days with standard deviation of 3. Mean APACHE II score was altogether higher in non-survivors (20) contrasted with 12 in non-survivors. P esteem was 0.002 . Middle mortality hazard was higher in non-survivors (40) contrasted with 15 in survivors. P esteem was 0.012 .

In the investigation of Naved et al., [5], in the primary gathering, there were 30 patients. Out of them, $27(90 \%)$ were released and 3 passed on $(10 \%)$. There were 100 patients in second gathering; 71 (71\%) were released and 29 (29\%) kicked the bucket. 83 patients were in gathering III, 33 patients $(39 \%)$ were released, while 50 patients $(61 \%)$ kicked the bucket. Gathering IV had 39 patients; $33(84.6 \%)$ kicked the bucket and just 6 $(15.4 \%)$ endure. There was just a single patient in gathering V, with APACHE II score of $>40$, and he passed on $(100 \%)$. This uncovered that there may be more odds of death in the event of high APACHE-II score $(p=0.001$, and more odds of getting out from the ICU if there should arise an occurrence of low APACHE. Mean ICU remain in the patients who terminated was $6.65( \pm 4.76$ going from 1 to 20$)$ days while in the individuals who endure and released was 7.34 ( \pm 7.01 going from 1 to 51$)$ days. This uncovered irrelevant distinction of mean ICU remain between the lapsed and alive patients $(\mathrm{p}=0.365)$. Irrelevant yet a backwards relationship $(r=-0.084, p<0.183)$ was seen between APACHE-II score and length of ICU remain.

The current examination indicated that cortisol demonstrated non-critical connections with other investigation boundaries. VMA demonstrated huge positive connection with APACHE II score $(r=0.395$ and $\mathrm{P}$ esteem $=0.012$ ). There were no huge relationships between's VMA and different boundaries. Middle Cortisol level was essentially higher in non-survivors (50) contrasted with survivors (20). P esteem was 0.042. Middle VMA was altogether higher in non-survivors (41) contrasted with survivors (10). P esteem was 0.021 .

Our outcomes were upheld by investigation of Tarjányi et al., (1) as they revealed that free cortisol was fundamentally higher in non-survivors at affirmation and 6, 24 and 48 hours after confirmation. Since intubation and mechanical ventilation may expand cortisol levels and might be related with terrible guess, the cortisol levels of ventilated and not ventilated patients were likewise looked at each time focuses. The death rate was likewise fundamentally raised in cases that necessary ventilation $(\mathrm{p}<0.001)$.

Balbão et al., [2] exhibited that there was a huge positive relationship between's serum complete cortisol and salivary cortisol focuses at benchmark $(\mathrm{r}=0 \bullet 67$; $\mathrm{P}<$ $0 \bullet 0001)$ and following ACTH $(\mathrm{r}=0 \bullet 41 ; \mathrm{P}=0 \bullet 02)$ in the fundamentally sick patients. Additionally, a critical backwards relationship was seen between salivary cortisol fixation post-ACTH and mean vasoactive inotropic score over the initial $48 \mathrm{~h}$ following PICU confirmation $(\mathrm{r}=-0 \cdot 56 ; \mathrm{P}=0 \bullet 0008)$.

In the examination in our grasp, there were no critical contrasts between survivors and non-survivors as respect age and sex. ROC examination was accomplished for cortisol expectation of mortality. It was discovered that AUC was 0.783 with $95 \%$ CI going from 0.625 to 0.940 . Best cutoff was $>29$ at which affectability and particularity were $100 \%$ and $60 \%$ separately.

Our outcomes were upheld by investigation of Tarjányi et al., [1], free cortisol was fundamentally associated with anticipated mortalities. A free cortisol of $28.2 \mathrm{nmol} 1-1$ at affirmation for instance had $88.2 \%$, affectability and $67.3 \%$ explicitness to foresee mortality. 
APACHE-II score. On the off chance that variable has not been estimated, it is allocated zero focuses. The factors are, inside temperature, pulse, mean blood vessel pressure, respiratory rate, oxygenation, blood vessel $\mathrm{pH}$, serum sodium, serum potassium, serum creatinine, hematocrit, white platelets tally and Glasgow unconsciousness scale. Second segment is age change: From one to six focuses are added for patients more established than 44 years old. Third part of APACHE-II is constant wellbeing assessment. An extra change is made for patients with extreme and persistent organ disappointment including the heart, lungs, kidneys, liver and safe framework [9].

As indicated by Karagüzel et al., (10), in patients with disease, cortisol levels were discovered to be connected with ACTH ( $\mathrm{p}=0.016, \mathrm{r}=0.394)$, IL-6 ( $\mathrm{p}=$ $0.012, r=0.408)$, and procalcitonin $(\mathrm{p}=0.005, \mathrm{r}=$ $0.456)$ levels. The patients with contamination had fundamentally higher TNF- $\alpha$ levels than non-irresistible patients $(\mathrm{p}=0.009)$.

Nonetheless, in the investigation of Riutta et al., [11], the seriousness of organ brokenness didn't relate either with the VMA discharge or with serum cortisol fixation.

Bonifačić et al., [12] uncovered that estimation of vanillylmandelic decidedly related with utilitarian result of ischaemic stroke. Positive results connected with diminished qualities, as opposed to expanded qualities, which were related with horrible results.

The cortisol reaction to health related crises is by all accounts corresponding to the seriousness of ailment. It very well may be assumed that the adrenal reaction is typically suitable and maximal in hazardous conditions. As per another idea of cortisol homeostasis in basic disease, ACTH isn't raised, even smothered because of the high cortisol level. It is conceivable that ACTH incitement test doesn't bring about huge cortisol height in those patients who as of now have high cortisol level. They are respected having relative adrenal inadequacy. The overproduction of cortisol is likewise discussed. The essential driver of cortisol height presumably is the diminished digestion of glucocorticoids [13].

\section{References}

[1] Z.Tarjanyi, G.Montsko, P.Kenyeres. free and total cortisol levels are useful prognostic markers in critically ill patients:a prospective observational study, european journal of endocrinology, Vol.171,PP.751-759,2014.
[2] V.M.P.Balbao, M.M.A.Costa, M.Castrot. evaluation of adrenal function in critically ill children, clinical endocrinology, Vol. 81,PP.559-565. ,2014.

[3] S.Sam, T.Corbridget, B.Mokhlesit. cortisol levels and mortality in sever sepsis, clinical endocrinology, Vol.60,PP.29-35,2004.

[4] D.Bonifacic, M.Aralica, V.Sotosek. values of vanillyl mandelic acid and homovanillic acid in the Urine as potential prognostic biomarkers in ischaemic stroke patients, biomarkers, Vol.22 (8),PP.790-797,2017.

[5] S.A.Naved, S.Siddiqui, F.H. Khan. APACHE-II score correlation with mortality and length of stay in an intensive care unit. Journal of the College of Physicians and Surgeons Pakistan, Vol.21(1),PP.4,2011.

[6] C.F.Pizarro, EJ.Troster. Adrenal function in sepsis and septic shock. J Pediatr (Rio J), Vol.83,PP.155$162,2007$.

[7] J.J.Ligtenberg, J.G.Zijlstra. Relative adrenal insuffiency syndrome. In: Vincent J-L, ed. Yearbook of Intensive Care and Emergency Medicine. Berlin Heidelberg: Springer-Verlag.vol.8,PP.492-498,2002.

[8] S.Barco, I.Gennai, G.Reggiardo. Urinary homovanillic and vanillylmandelic acid in the diagnosis of neuroblastoma: report from the Italian Cooperative Group for Neuroblastoma. Clin Biochem.vol.47,PP.848-852,2014.

[9] L.A.Dossett, L.A.Redhage, R.G.Sawyer, A.K.May. Revisiting the validity of APACHE-II in the trauma ICU: improved risk stratification in critically injured adults. Injury.vol.40,PP.993-8. Epub 2009 Jun $16,2009$.

[10] G.Karagüzel, S.Atay, O.Değer. The effects of three specific conditions related to critical care on adrenal function in children. Intensive care medicine, Vol.38(10),PP.1689-1696,2012.

[11]A.Riutta, P.Ylitalo, S.Kaukinen. Diurnal variation of melatonin and cortisol is maintained in non-septic intensive care patients. Intensive care medicine.vol.35(10),PP.1720,2009.

[12]D.Bonifačić, M.Aralica, V.Sotošek Tokmadžić. Values of vanillylmandelic acid and homovanillic acid in the urine as potential prognostic biomarkers in ischaemic stroke patients. Biomarkers.vol.22(8),PP.790-797,2017.

[13] G.Van den Berghe. Endocrine evaluation of patients with critical illness. Endocrinology and Metabolism Clinics of North America, Vol.32,PP.385-410,2003. 